\title{
Momentum-dependent potential and collective flows within the relativistic quantum molecular dynamics approach based on relativistic mean-field theory
}

\author{
Yasushi Nara, ${ }^{1,2}$ Tomoyuki Maruyama, ${ }^{3}$ and Horst Stoecker ${ }^{2,4,5}$ \\ ${ }^{1}$ Akita International University, Yuwa, Akita-city 010-1292, Japan \\ ${ }^{2}$ Frankfurt Institute for Advanced Studies, D-60438 Frankfurt am Main, Germany \\ ${ }^{3}$ College of Bioresource Sciences, Nihon University, Fujisawa 252-0880, Japan \\ ${ }^{4}$ Institut für Theoretische Physik, Johann Wolfgang Goethe Universität, D-60438 Frankfurt am Main, Germany \\ ${ }^{5}$ GSI Helmholtzzentrum für Schwerionenforschung GmbH, D-64291 Darmstadt, Germany
}

(Dated: September 1, 2020)

\begin{abstract}
Relativistic quantum molecular dynamics based on the relativistic mean field theory (RQMD.RMF) is extended by including momentum-dependent potential. The equation of state (EoS) dependence of the directed and the elliptic flow of protons in the beam energy range of $2.3<\sqrt{s_{N N}}<20 \mathrm{GeV}$ is examined. It is found that the directed flow depends strongly on the optical potential at high energies, $\sqrt{s_{N N}}>3 \mathrm{GeV}$, where no information is available experimentally. The correlation between effective mass at saturation density and the optical potential is found: smaller values of effective mass require smaller strengths of the optical potential to describe the directed flow data. This correlation can also be seen in the beam energy dependence of the elliptic flow at $\sqrt{s_{N N}}>3 \mathrm{GeV}$, although its effect is rather weak. On the other hand, stiff EoS is required to describe the elliptic flow at lower energies. Experimental constraints on the optical potential from $p A$ collisions will provide important information on the EoS at high energies. The proton directed and the elliptic flow are well described in the RQMD.RMF model from $\sqrt{s_{N N}}=2.3$ to $8.8 \mathrm{GeV}$. In contrast, to reproduce the collapse of the directed flow above $10 \mathrm{GeV}$, pressure has to be reduced, which indicates a softening of the EoS around $\sqrt{s_{N N}}=10 \mathrm{GeV}$.
\end{abstract}

PACS numbers: $25.75 .-\mathrm{q}, 25.75 . \mathrm{Ld}, 25.75 . \mathrm{Nq}, 21.65 .+\mathrm{f}$

\section{INTRODUCTION}

High energy heavy-ion collisions provide a unique opportunity to explore the properties of strongly interacting QCD matter for a wide range of temperatures and densities. In particular, collisions in the energy range of $2<\sqrt{s_{N N}}<20$ $\mathrm{GeV}$ create high baryon density matter, and should be the best place to search for the onset of a phase transition as well as critical point in QCD matter. The ongoing beam energy scan program (BES) [1, 2] at the BNL-RHIC-STAR- and the CERN-SPS-NA49 and -NA61/SHINE experiments [3] have measured beam energy, collision system, and centrality dependence of observables such as collective flows, fluctuations of conserved charges, which are expected to be sensitive to a phase transition and/or critical point. Future experiments, such as RHIC-BESII [4], STAR FXT, CBM and HADES at FAIR [5, 6], BM@N and MPD at NICA [7], HIAF at Canton, as well as the proposed J-PARC-HI [8], will further offer excellent opportunity to explore the highest density baryonic matter sector of QCD, and determine the phase structure of QCD with high statistics data.

To extract the information on the properties of high dense QCD matter from heavy-ion experimental data, details of the collision dynamics have to be understood. For this purpose, the transport models such as non-equilibrium microscopic transport models [9-14], hydrodynamical models [15], and hybrid models [16, 18, 23] have been used to simulate space-time evolutions of hot and dense matter created in nuclear collisions at high baryon density regions. It has been argued for a long time the determination of the equation of state (EoS) from collective flows such as directed as well as elliptic flow, as they are sensitive to the EoS [24-31]. For instance, fluid dynamics predicts negative directed flow of protons at the vicinity of the softest point in the EoS with a first-order phase transition [32 -36], which is also confirmed by microscopic transport model calculations [37,-39]. The NA49 [40] and the STAR [41, 42] collaborations discovered the negative proton directed flow at $\sqrt{s_{N N}}>8 \mathrm{GeV}$, which locates rather higher beam energies than the AGS energies that is expected to be a softest point by most of the theoretical predictions.

The time evolution of heavy-ion collisions generally consist of far from an equilibrium state to late possible equilibrium stage followed by a freeze-out process. Non-equilibrium microscopic transport approach is a theoretical framework to simulate a collision of nuclei from initial to final stages in a unified way, and it has been widely used to describe nuclear collisions from low to high energies, see Ref [43] for the recent comparison of heavy-ion transport codes. A relativistic transport approach based on the relativistic meanfield theory of Walecka type, called relativistic BoltzmannUehling-Uhlenbeck (RBUU) has been formulated, and several transport codes have been developed for heavy-ion collisions [14, 44-48]. On the other hand, the quantum molecular dynamics (QMD) [11, 12, 49, 50] is an $N$-body approach, which simulates multi-particle collision dynamics beyond the time evolution of one-particle distribution function like RBUU models. Therefore, the QMD model can be applied to study, for example, multi-fragmentations and eventby-event fluctuations. The relativistic version of the QMD model (RQMD) has been developed by the Lorentz scalar treatment of the Skyrme potential [9, 10, 51-54]. RQMD with the relativistic mean-field has been developed in Ref. [55] for the intermediate energy heavy-ion collisions up to $E_{\text {lab }}=2 \mathrm{~A}$ $\mathrm{GeV}$. Recently, RQMD based on the relativistic mean-field 
theory (RQMD.RMF) [56] has been implemented into the transport code JAM [57] to simulate high energy nuclear collisions. It is shown that this relativistic transport approach RQMD.RMF reproduces the beam energy dependence of the directed as well as the elliptic flow from $\sqrt{s_{N N}}=2.4 \mathrm{GeV}$ up to $8 \mathrm{GeV}$.

The importance of the momentum dependence of the meanfield was realized for the extraction of the EoS from heavyion collisions [58, 59]. An extension of the RBUU model by introducing an additional momentum-dependent potential has been formulated in Ref. [60], which remedies the problem of too repulsive potential in the Walecka model. Numerical simulations of RBUU with momentum-dependent interaction was performed at low and intermediate heavy-ion collisions at $E_{\text {lab }}<2 A \mathrm{GeV}$ within polynomial approximation for the momentum-dependent potentials [61, 62]. RBUU approach with momentum-dependent scalar and vector form factor was applied for the study of the beam energy dependence of the directed and the elliptic flow [63].

RQMD approach with relativistic mean-field including explicit momentum-dependent interactions have not been developed to date. In this paper, we extend our RQMD.RMF approach [56] by incorporating momentum-dependent potential in line with Ref. [60], and apply it to high energy heavy-ion collisions at $2.3 \lesssim \sqrt{s_{N N}} \lesssim 20 \mathrm{GeV}\left(1 \lesssim E_{\text {lab }} \lesssim 160 A\right.$ $\mathrm{GeV})$ to investigate the effects of momentum-dependence on the collective flow. Optical potential has been extracted by experiments up to the beam energy of $E_{\text {lab }}=1 \mathrm{GeV}$. Therefore, we test different strengths of the optical potential at $E_{\text {lab }}>1$ $\mathrm{GeV}$ to study the sensitivity of the flows to the optical potential.

This paper is organized as follows: Section II describes the nonlinear $\sigma-\omega$ model with momentum-dependent potential and its implementation into the RQMD framework. Section III presents the results for the beam energy dependence of the directed and the elliptic flow, as well as the rapidity dependence of the directed flow. The summary is given in Sec. IV.

\section{MODEL}

We first present our EoS from relativistic mean-field theory with momentum-dependent potentials, and then describe how to implement it into the framework of RQMD approach. This approach has been realized in the transport code JAM, which enables us to simulate nuclear collisions at high energies.

\section{A. EoS from the relativistic mean-field with momentum-dependent potential}

A covariant treatment of the momentum dependence of the relativistic potentials in the relativistic mean-field theory was formulated in Ref. [60]. Here we shall employ a Lorentzian form of momentum-dependent potential which depends only on the spacial part of momentum neglecting the energy dependence, which is related to nonlocality in time. This is consistent with our assumption of time-fixation conditions specified below in our RQMD approach. Thus, we introduce the following momentum-dependent scalar and vector potential:

$$
\begin{aligned}
V_{s}^{\mathrm{MD}} & =\frac{\bar{g}_{s}^{2}}{m_{s}^{2}} \int d^{3} p \frac{m^{*}}{p_{0}^{*}} \frac{f(x, p)}{1+\left(\boldsymbol{p}-\boldsymbol{p}^{\prime}\right)^{2} / \Lambda_{s}^{2}}, \\
V_{\mu}^{\mathrm{MD}} & =\frac{\bar{g}_{v}^{2}}{m_{v}^{2}} \int d^{3} p \frac{p_{\mu}^{*}}{p_{0}^{*}} \frac{f(x, p)}{1+\left(\boldsymbol{p}-\boldsymbol{p}^{\prime}\right)^{2} / \Lambda_{v}^{2}},
\end{aligned}
$$

where $f(x, p)$ is a phase space distribution function. At zero temperature, it is given by

$$
f(x, p)=\frac{g_{N}}{(2 \pi)^{3}} \theta\left(p_{F}-|\boldsymbol{p}|\right) .
$$

where $p_{F}$ is the Fermi momentum, and $g_{N}=4$ is the degeneracy factor for spin and isospin of nucleons. In the actual implementation into the RQMD model, the arguments of the momentum-dependent potentials are replaced by the relative momentum in the two-body center-of-mass frame between interacting particles to maintain the covariance of the theory.

The energy density for nuclear matter in the relativistic mean-field theory with $\sigma$ - and $\omega$ - meson-baryon interactions with momentum-dependent potentials is given by [60]

$$
\begin{aligned}
e & =\int d^{3} p p_{0} f(p)+U(\sigma) \\
& +\frac{1}{2} \int \frac{d^{3} p}{p_{0}^{*}}\left(m^{*} V_{s}^{\mathrm{MD}}-p^{* \mu} V_{\mu}\right) f(p) .
\end{aligned}
$$

Here, the vacuum mass $m$ and canonical momentum $p_{\mu}$ are modified by the scalar potential $S$ and the vector potential $V_{\mu}$, which define the effective mass $m^{*}$ and kinetic momentum $p^{*}$, respectively:

$$
\begin{aligned}
m^{*} & =m-S=m-g_{s} \sigma-V_{s}^{\mathrm{MD}}, \\
p_{\mu}^{*} & =p_{\mu}-V_{\mu}=p_{\mu}-g_{v} \omega_{\mu}-V_{\mu}^{\mathrm{MD}} .
\end{aligned}
$$

The mass-shell constraint $p^{* 2}-m^{* 2}=0$ is consistent with the single-particle energy as

$$
p_{0}=\sqrt{m^{* 2}+\boldsymbol{p}^{* 2}}+g_{v} \omega_{0}+V_{0}^{\mathrm{MD}} .
$$

For the scalar field, the following nonlinear self-interaction is introduced [64]:

$$
U(\sigma)=\frac{m_{\sigma}^{2}}{2} \sigma^{2}+\frac{g_{2}}{3} \sigma^{3}+\frac{g_{3}}{4} \sigma^{4} .
$$

The $\sigma$ and $\omega$ field are obtained by solving the self-consistent equations

$$
m_{s}^{2} \sigma+g_{2} \sigma^{2}+g_{3} \sigma^{3}=g_{s} \rho_{s}, m_{v}^{2} \omega^{0}=g_{v} \rho_{v} .
$$

Here $\rho_{s}=\int d^{3} p \frac{m^{*}}{p_{0}^{*}} f(p)$ is the scalar density, and $\rho_{v}=$ $\int d^{3} p f(p)$ is a zeroth component of the vector density.

In order to fix the parameters in the momentum-dependent potentials, we fit the real part of the experimentally determined nucleon-nucleus optical potential [65] together with the binding energy per nucleon $E / A=p_{0}\left(p_{F}\right)-m_{N}=16 \mathrm{MeV}$. 
TABLE I. Parameters for the relativistic mean-field theory with nonlinear scalar interaction and momentum-dependent potentials. A binding energy of $E / A=-16 \mathrm{MeV}$ at normal nuclear matter density of $\rho_{0}=0.1681 / \mathrm{fm}^{3}$, a $\sigma$ mass of $m_{s}=0.55 \mathrm{GeV}$, and an $\omega$ mass of $m_{v}=0.783 \mathrm{GeV}$ are used.

\begin{tabular}{ccccccccc}
\hline \hline \multicolumn{1}{c}{} & & NS1 & NS2 & NS3 & MD1 & MD2 & MD3 & MD4 \\
\hline$K$ & $(\mathrm{MeV})$ & 380 & 210 & 380 & 380 & 380 & 380 & 210 \\
$m^{*} / m$ & & 0.83 & 0.83 & 0.7 & 0.65 & 0.65 & 0.65 & 0.83 \\
$U_{\text {opt }}(\infty)$ & $(\mathrm{MeV})$ & 95 & 98 & 200 & 95 & 30 & -0.4 & 67 \\
$g_{s}$ & & 6.448 & 7.902 & 8.864 & 9.030 & 9.233 & 5.439 & 4.059 \\
$g_{v}$ & & 6.859 & 6.859 & 10.07 & 6.740 & 3.888 & 0.0 & 5.632 \\
$g_{2}$ & $(1 / \mathrm{fm})$ & -38.0 & 44.31 & 2.191 & 4.218 & 4.012 & -15.59 & -160.3 \\
$g_{3}$ & & 339.6 & 21.99 & 27.07 & 6.667 & 5.520 & 391.9 & 2684 \\
$\bar{g}_{s}$ & & - & - & - & 3.186 & 2.502 & 7.711 & 5.544 \\
$\bar{g}_{v}$ & & - & - & - & 8.896 & 10.43 & 11.22 & 3.926 \\
$\Lambda_{s}$ & $(\mathrm{GeV})$ & - & - & - & 0.641 & 0.4897 & 1.702 & 0.704 \\
$\Lambda_{v}$ & $(\mathrm{GeV})$ & - & - & - & 1.841 & 2.489 & 1.898 & 4.252 \\
\hline \hline
\end{tabular}

We define the optical potential by subtracting kinetic energy from the single-particle energy of nucleon [66-68]

$$
U_{\text {opt }}(p)=p_{0}(p)-\sqrt{m_{N}^{2}+\boldsymbol{p}^{2}},
$$

where $m_{N}$ is the free nucleon mass. This optical potential is similar to the Schrödinger-equivalent potential at low to moderate momenta, but it approaches a constant value at highenergy limit in contrast to the Schrödinger-equivalent potential that linearly depends on the kinetic energy for nonzero vector potential. Remaining parameters of the EoS are determined by the condition that ground state is a minimum in the EoS at the normal nuclear matter density $\rho_{B}=\rho_{0}=0.168$ $1 / \mathrm{fm}^{3}: P=\rho_{B}^{2} \partial\left(e / \rho_{B}\right) /\left.\partial \rho_{B}\right|_{\rho_{B}=\rho_{0}}=0$ for a given incompressibility $K=9 \rho_{B}^{2} \partial^{2}\left(e / \rho_{B}\right) /\left.\partial \rho_{B}^{2}\right|_{\rho_{B}=\rho_{0}}$ and effective nucleon mass $m^{*}\left(\rho_{0}\right)$ at the normal nuclear matter density. The parameter sets are given in Table I for different incompressibilities, effective masses, and optical potentials to investigate the influence of EoS on the collective flows.

In the upper panel of Fig. 1. we compare energy dependence of the optical potential defined by Eq. (10) at normal nuclear matter density with the parameters with and without momentum-dependence. The parameter set NS3 $\left(\mathrm{m}^{*} / \mathrm{m}=\right.$ $0.7)$ reproduces the experimentally determined optical potential [65] up to $E_{\text {lab }}=0.5 \mathrm{GeV}$, while NS1 $\left(\mathrm{m}^{*} / m=0.83\right)$ significantly underestimates the data. In contrast, the optical potential of NS3 has much higher values than the data at higher beam energies above $E_{\text {lab }}=1 \mathrm{GeV}$, as is well known that the Walecka type model has strong energy dependence. Analysis of the directed flow data by the transport models with $\sigma-\omega$ interactions found that the parameter set with $m^{*} / m \approx 0.7$ fits the flow data at lower beam energies $E_{\text {lab }}<0.4 A \mathrm{GeV}$, while the parameter set with $m^{*} / m \approx 0.83$ is favored by the data above $E_{\text {lab }} \approx 0.8 A \mathrm{GeV}$ [46, 47, 55, 56]. This fact indicates that the values of the optical potential may be close to those in the parameter set NS1 at $E_{\text {lab }} \geq 1 \mathrm{GeV}$. Based on this observation, the parameter
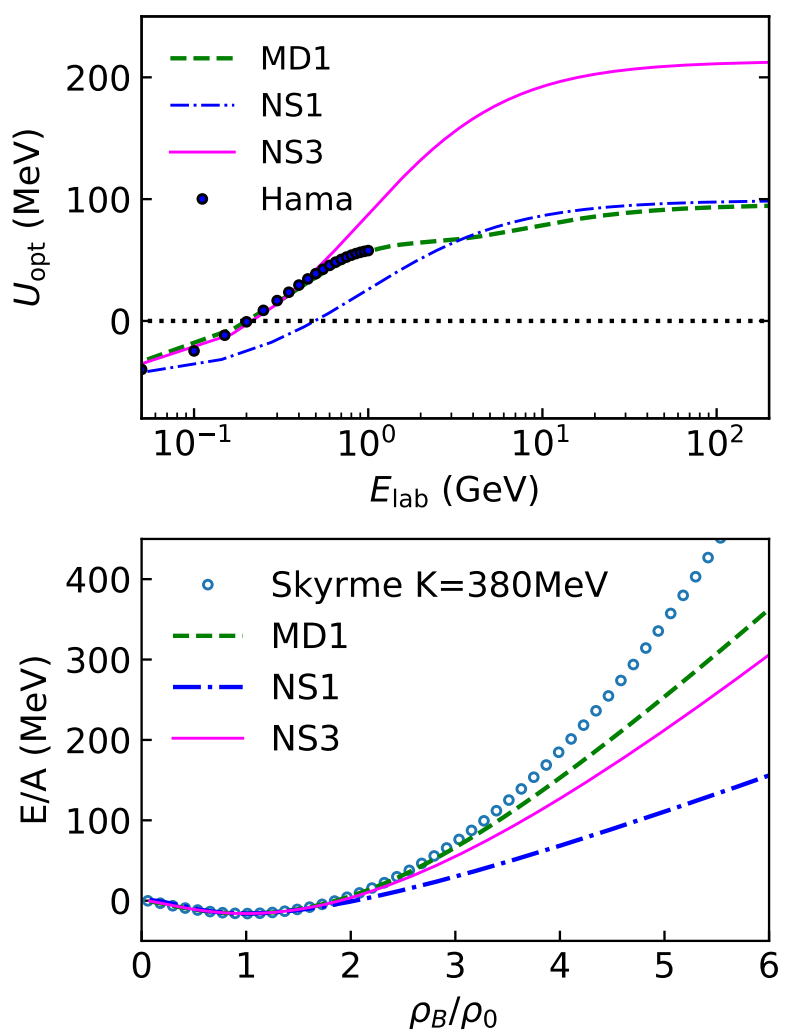

FIG. 1. Upper panel: optical potentials in normal nuclear matter density as a function of incident energy. Lower panel: total energy per nucleon as a function of the normalized baryon density at zero temperature. The dashed, dot-dashed, and solid lines show the results for the parameter set MD1, NS1, and NS3, respectively. The hard EoS from Skyrme potential with $K=380 \mathrm{MeV}$ is shown by open circles. The full circles correspond to the results of the global Dirac optical model fit to $p$-nucleus elastic scattering data by Hama et al. [65].

set MD1 is obtained by assuming values of optical potential similar to those in the NS1 parameter set at $E_{\text {lab }}>1 \mathrm{GeV}$ as shown in the dashed line in Fig. 1. Its asymptotic value is $U_{\text {opt }}(\infty)=95 \mathrm{MeV}$ as indicated in Table II The lower panel of Fig. 1 1 compares the energy per nucleon at zero temperature

$$
\frac{E}{A}=\frac{e}{\rho_{B}}-m_{N}
$$

as a function of baryon density for different parameter sets. As is well known, stiffness of the EoS with respect to baryon density is mainly determined by the value of the effective mass; smaller effective mass yields stiffer EoS. Baryon-density dependence in the MD1 parameter set is similar to the one in the set NS3 which overestimates the flow data, while NS1, which reproduces the flow data, is softer than NS3 in terms of the baryon density as NS1 has a larger effective mass. As a comparison, hard $\operatorname{EoS}(K=380 \mathrm{MeV})$ from the nonrelativistic Skyrme potential

$$
V_{\mathrm{sk}}=\alpha \rho_{B}+\beta \rho_{B}^{\gamma}
$$


is plotted. The transport models with this Skyrme hard EoS reproduce the elliptic flow data at $E_{\text {lab }}<10 A \mathrm{GeV}$ [69, 70].
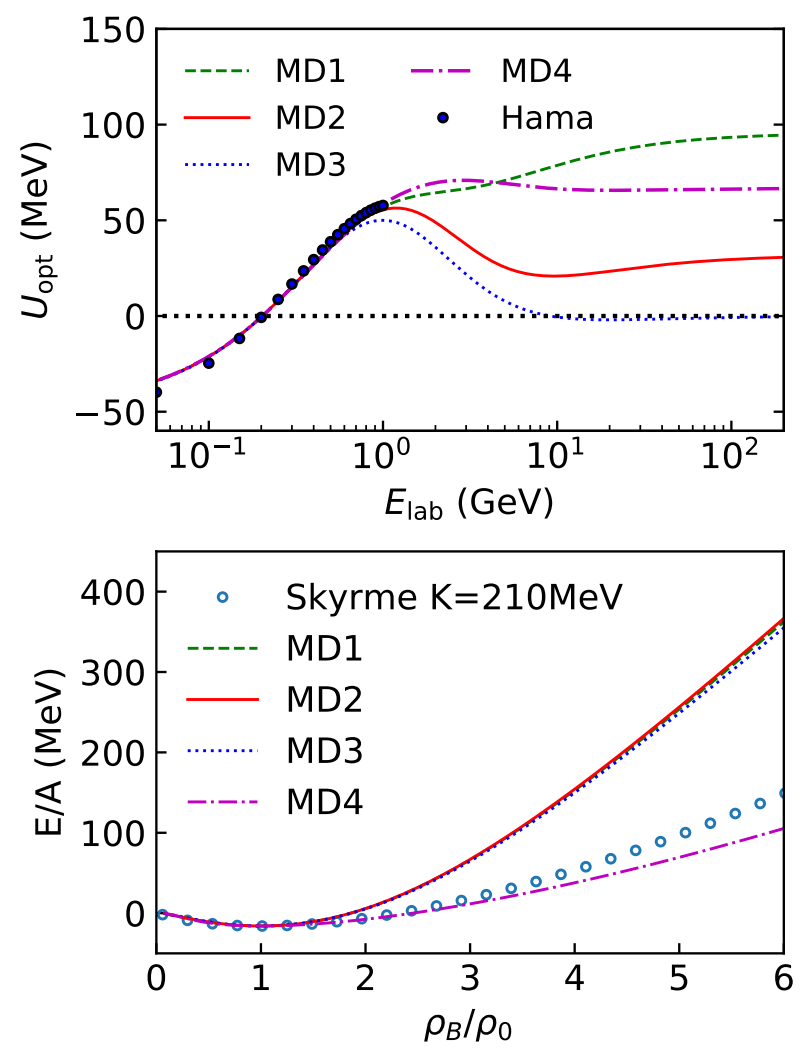

FIG. 2. Same as Fig. 1. but for different parameter sets. The dashed, solid, dotted, and dot-dashed lines show the results for the parameter set MD1, MD2, MD3, and MD4, respectively. The soft EoS from Skyrme potential with $K=210 \mathrm{MeV}$ is also shown by open circles.

There is no experimental information on the nucleon optical potential at higher energies above $E_{\text {lab }}=1 \mathrm{GeV}$. Therefore, we consider different values of optical potential at high energy limit as shown in the upper panel of Fig. 2, keeping the same baryon density dependence (MD1, MD2, and MD3) assuming the same effective mass $m^{*} / m=0.65$ and incompressibility $K=380 \mathrm{MeV}$ (see the lower panel of Fig. 2). We also prepare the soft EoS MD4 ( $K=210 \mathrm{MeV}$ and $\left.m^{*} / m=0.83\right)$, but its optical potential is almost flat at $E_{\text {lab }}>1 \mathrm{GeV}$. The baryon density dependence of the MD4 parameter set is as soft as that of the non-relativistic Skyrme type potential with $K=210 \mathrm{MeV}$ as shown in the lower panel of Fig.2.

\section{B. Relativistic quantum molecular dynamics}

We implement the relativistic EoS constructed above into the microscopic $N$-body non-equilibrium transport approach RQMD [9] which is formulated based on the constraint Hamiltonian dynamics [71]. The manifestly covariant formulation for the $N$-body dynamics uses $8 N$ four-vectors $q_{i}^{\mu}$ and $p_{i}^{\mu}(i=1, \ldots, N)$ for the position and momentum coordi- nates of particles, respectively. Thus, $2 N$ constraints are employed to reduce the number of dimensions from $8 N$ to the physical $6 N$,

$$
\phi_{i} \approx 0,(i=1, \ldots, 2 N)
$$

where the sign $\approx$ stands for Dirac's weak equality: this equality has to be satisfied on the physical $6 N$ phase space. $2 N-1$ constraints should be Poincaré invariant, while the $2 N$ th is not necessarily Poincaré invariant, since it determines the evolution parameter $\tau$. The Hamiltonian of the $N$-body system is constructed as the linear combination of $2 N-1$ constraints

$$
H=\sum_{j=1}^{2 N-1} u_{j}(\tau) \phi_{j}
$$

with the Lagrange multipliers $u_{j}(\tau)$. The equations of motion are then given by

$$
\begin{aligned}
& \frac{d q_{i}}{d \tau}=\left[H, q_{i}\right] \approx \sum_{j=1}^{2 N-1} u_{j} \frac{\partial \phi_{j}}{\partial p_{i}} \\
& \frac{d p_{i}}{d \tau}=\left[H, p_{i}\right] \approx-\sum_{j=1}^{2 N-1} u_{j} \frac{\partial \phi_{j}}{\partial q_{i}}
\end{aligned}
$$

where the Poisson brackets are defined as

$$
[A, B]=\sum_{k}\left(\frac{\partial A}{\partial p_{k}} \cdot \frac{\partial B}{\partial q_{k}}-\frac{\partial A}{\partial q_{k}} \cdot \frac{\partial B}{\partial p_{k}}\right) .
$$

We require that the constraints are conserved in time:

$$
\frac{d \phi_{i}}{d \tau}=\frac{\partial \phi_{i}}{\partial \tau}+\left[H, \phi_{i}\right] \approx 0
$$

As $2 N-1$ constraints do not depend explicitly on $\tau$, the Lagrange multipliers $u_{i}$ are solved as

$$
u_{i} \approx-\frac{\partial \phi_{2 N}}{\partial \tau} C_{2 N, i},(i=1, \cdots, 2 N-1),
$$

where $C_{i j}^{-1}=\left[\phi_{i}, \phi_{j}\right]$. In this way, the equations of motion Eq. (15) and the Lagrange multipliers Eq. (18) uniquely determine the trajectory of the coupled system of particles in $6 \mathrm{~N}$ phase space.

For our relativistic scalar and vector interaction in the RQMD.RMF approach, we choose the $N$ on-mass shell conditions

$$
\phi_{i} \equiv p_{i}^{* 2}-m_{i}^{* 2}=\left(p_{i}-V_{i}\right)^{2}-\left(m_{i}-S_{i}\right)^{2},(i=1, \ldots, N)
$$

for the $i$ th particle, where $V_{i}^{\mu}$ and $S_{i}$ are the single-particle vector and scalar potential. The remaining $N$ constraints fix the time of $N$ particles. Here we use the same time fixation constraints proposed in Ref. [52, 72], which equate the all time coordinates of particles in the reference frame:

$$
\begin{aligned}
\phi_{i+N} & \equiv \hat{a} \cdot\left(q_{i}-q_{N}\right), \quad(i=1, \cdots, N-1), \\
\phi_{2 N} & \equiv \hat{a} \cdot q_{N}-\tau,
\end{aligned}
$$


where $\hat{a}$ is a unit-four-vector $\hat{a}=(1, \mathbf{0})$ in the reference frame [52]. A convenient choice may be $\hat{a}=P / \sqrt{P^{2}}$ with $P=\sum_{i}^{N} p_{i}$, which equates the time coordinates of all particles in the overall center-of-mass system [72].

We further make assumption that the arguments of the potentials are replaced by the free ones [52]. Then, the equations of motion for the $i$ th particle are obtained as

$$
\begin{aligned}
\dot{\boldsymbol{x}}_{i} & =\frac{\boldsymbol{p}_{i}^{*}}{p_{i}^{* 0}}+\sum_{j=1}^{N}\left(\frac{m_{j}^{*}}{p_{j}^{* 0}} \frac{\partial m_{j}^{*}}{\partial \boldsymbol{p}_{i}}+v_{j}^{*} \cdot \frac{\partial V_{j}}{\partial \boldsymbol{p}_{i}}\right), \\
\dot{\boldsymbol{p}}_{i} & =-\sum_{j=1}^{N}\left(\frac{m_{j}^{*}}{p_{j}^{* 0}} \frac{\partial m_{j}^{*}}{\partial \boldsymbol{r}_{i}}+v_{j}^{*} \cdot \frac{\partial V_{j}}{\partial \boldsymbol{r}_{i}}\right),
\end{aligned}
$$

where $v_{i}^{* \mu}=p_{i}^{* \mu} / p_{i}^{* 0}$.

Within the RQMD approach, the scalar density and baryon current are evaluated by employing the Gaussian wave packet:

$$
\rho_{s, i}=\sum_{j \neq i} \frac{m_{j}}{p_{j}^{0}} \rho_{i j}, \quad J_{i}^{\mu}=\sum_{j \neq i} B_{j} \frac{p_{j}^{\mu}}{p_{j}^{0}} \rho_{i j} .
$$

Here $B_{j}$ is a baryon number of the $j$ th particle. Note that we use a free mass $m_{j}$ and canonical momentum $p_{j}^{\mu}$ to compute the scaler density and the baryon current, since we assumed that the arguments of potentials are replaced by the free ones in the derivation of the equations of motion. However, we found that even though the scalar density and the baryon current are defined by using effective mass $m_{i}^{*}$ and kinetic momentum $p_{i}^{* \mu}$, numerical results turn out to be almost unchanged. The Gaussian $\rho_{i j}$ is given by

$$
\rho_{i j}=\frac{\gamma_{i j}}{(2 \pi L)^{3 / 2}} \exp \left(q_{T, i j}^{2} / 2 L\right)
$$

where $q_{T, i j}^{2}$ is a distance squared measured in a certain frame, and $\gamma_{i j}$ is a Lorentz $\gamma$ factor which ensures the correct normalization of the Gaussian [73] in Eq. 23]. We note that this definition of $\rho_{i j}$ is different from the so-called interaction density by a factor of two which is defined by the overlap of density with other Gaussian wave packets in the QMD approach with Skyrme force. Throughout this work, the Gaussian width is fixed at $L=1.0 \mathrm{fm}^{2}$.

There are several choices for the reference frame to define a Lorentz invariant distance squared $q_{T i j}^{2}$ :

(1) overall center-of-mass frame,

(2) center-of-mass frame between particle $i$ and $j$,

(3) rest frame of $j$ th particle.

Two-body c.m. frame has been used in most of the RQMD approach, while overall center-of-mass frame is used in Ref. [72], which is convenient when simulations are performed in the overall center-of-mass frame or box simulations, since the relative distance $q_{T, i j}$ becomes identical to the non-relativistic distance. This choice would be justified as far as the Gaussian width parameter $L$ is less than the order of the initial Lorentz contraction of the colliding two-nuclei.
The rest frame of particle is employed in the RLV model [48] to define the distance:

$$
q_{T, i j}=q_{i j}-\left(q_{i j} \cdot u_{j}\right) u_{j},
$$

where $q_{i j}=q_{i}-q_{j}$ and $u_{j}=p_{j} / m_{j}$. For this choice, the $\gamma$ factor in the Gaussian becomes $\gamma_{i j}=p_{j}^{0} / m_{j}$ which cancels the factor in the scalar density in Eq.(22), and the scalar density is explicitly Lorentz scalar:

$$
\rho_{s, i}=\sum_{j \neq i} \frac{1}{(2 \pi L)^{3 / 2}} \exp \left(\frac{q_{i j}^{2}-\left(q_{i j} \cdot u_{j}\right)^{2}}{2 L}\right) .
$$

We have checked that all of three choices yield practically identical results.

In RQMD.RMF with momentum-dependent potential, the single particle scalar and vector potential for $i$ th particle are defined as

$$
S_{i}=\frac{1}{2} g_{s} \sigma_{i}+V_{s, i}^{\mathrm{MD}}, \quad V_{i, \mu}=\frac{B_{i}}{2} g_{v} \omega_{i, \mu}+B_{i} V_{i, \mu}^{\mathrm{MD}} .
$$

Here the momentum-dependent potentials are given by

$$
\begin{aligned}
V_{s, i}^{\mathrm{MD}} & =\frac{1}{2} \frac{\bar{g}_{s}^{2}}{m_{s}^{2}} \sum_{i \neq j}^{N} \frac{m_{j}}{p_{j}^{0}} \frac{\rho_{i j}}{1-p_{T, i j}^{2} / \Lambda_{s}^{2}}, \\
V_{\mu, i}^{\mathrm{MD}} & =\frac{1}{2} \frac{\bar{g}_{v}^{2}}{m_{v}^{2}} \sum_{i \neq j}^{N} \frac{p_{\mu, j}}{p_{j}^{0}} \frac{B_{j} \rho_{i j}}{1-p_{T, i j}^{2} / \Lambda_{v}^{2}},
\end{aligned}
$$

where $p_{T, i j}$ is a relative momentum between $i$ th and $j$ th particle in the two-body center-of-mass frame:

$$
p_{T, i j}=p_{i j}-\frac{\left(p_{i j} \cdot P_{i j}\right)}{P_{i j}^{2}} P_{i j}
$$

where $p_{i j}=p_{i}-p_{j}$ and $P_{i j}=p_{i}+p_{j}$.

In the actual simulations, the non-linear $\sigma$-field, as well as the $\omega$-field at $i$ th particle's position, is evaluated by using a local density approximation [14, 44, 45], which neglects the derivatives of the scalar and the vector meson field:

$$
m_{s}^{2} \sigma_{i}+g_{2} \sigma_{i}^{2}+g_{3} \sigma_{i}^{3}=g_{s} \rho_{s, i}, \quad m_{v}^{2} \omega_{i}^{\mu}=g_{v} J_{i}^{\mu} .
$$

This approximation is widely applied to the simulations of high energy nuclear collisions [74-76]. See Ref. [77] for the study of the effects of the meson field radiation and retardation effects within the RBUU approach.

\section{Collision term}

Mean-field propagation by the Hamiltonian is combined with Boltzmann type collision term. Two-body collision terms are applied by the Monte Carlo method to simulate particle productions as well as decays by using the transport code JAM. Particle productions are modeled by the excitation of hadronic resonances and strings followed by their decays. A detailed discussion of the collision term treatment is found in 
Refs. [57, 78]. In JAM, free cross sections are used in the two-body collisions. In order to take into account in-medium threshold effects in two-body collisions, we evaluate the cross section with

$$
\sqrt{s_{\text {free }}}=\sqrt{s^{*}}-\left(m_{1}^{*}-m_{1}\right)-\left(m_{2}^{*}-m_{2}\right),
$$

where $m_{i}$ and $m_{i}^{*}$ are the free and effective hadron mass, respectively, and $s^{*}=\left(p_{1}^{*}+p_{2}^{*}\right)^{2}$, as employed in the RBUU calculations [14].

Collision term changes the momentum of particles, thus breaks energy conservation if momentum-dependent potentials are included, although total momentum is strictly conserved at each collision. However, the violation of energy conservation is found to be about 3-5\% level when momentumdependent potentials are included. We have checked the effects of the energy conservation by recovering total energy as follows by using the same method in Ref. [79]: First, go to the center-of-mass frame, then all momenta of the particles are scaled with the same factor $a$ such that $E_{\text {tot }}=$ $\sum_{i}\left(\sqrt{m_{i}^{* 2}+\left(a \boldsymbol{p}_{i}^{*}\right)^{2}}+V_{i}^{0}\right)$, where $E_{\text {tot }}$ is the total energy that we need to recover. We obtain the factor $a$ by the iteration:

$$
a^{\prime}=\frac{a E_{\mathrm{tot}}}{\sum_{i}\left(\sqrt{m_{i}^{* 2}+\left(a \boldsymbol{p}_{i}^{*}\right)^{2}}+V_{i}^{0}\right)}
$$

until the desired accuracy is achieved. It is expected that this procedure has little effect on the flows, since only the magnitude of momenta is iterated. Energy conservation is recovered at each two-body collision in the JQMD model [53], which employs a different algorithm than the one used in JAM for the treatment of collision term and decay. In this case, however, we have to update the collision list of all particles in JAM. In order to avoid this complication, we recover total energy at each Hamiltonian time step where the collision list of all particles has to be updated. This should be a good approximation, if time step is small and the number of collisions or decay is not so large within each time step. We have checked that, when energy conservation is recovered until it reaches within $0.1 \%$ accuracy, we still get the same results. In order to save computational time, all results in this paper are obtained without this option.

\section{RESULTS}

In this section, we present the results for sideward, directed, and elliptic flow in midcentral $\mathrm{Au}+\mathrm{Au}$ and $\mathrm{Pb}+\mathrm{Pb}$ collisions from the RQMD.RMF model using different equations of state described above.

The centrality cuts in the experimental data usually refer to cuts in the measured multiplicity distributions. The centrality cuts in the calculations are usually done by the analogous cuts in the impact parameter distributions. In the present paper, directed flow $\left(v_{1}\right)$ and elliptic flow $\left(v_{2}\right)$ are analyzed at midcentral collisions, which correspond to the impact parameter range (and multiplicity range) where the $v_{1}$ flow is rather close to its maximum. The E895 collaboration [80, 81] finds that their multiplicity- selected centrality class corresponds to impact parameters between 5 and $7 \mathrm{fm}$. The STAR FXT collaboration [82] selects, at $\sqrt{s_{N N}}=4.5 \mathrm{GeV}$, their centrality cut so that it comes close to the E895 one. Then the impact parameter range 5 to $7 \mathrm{fm}$ corresponds to the $10-25 \%$ midcentral multiplicity cut in the STAR experiment. The STAR collaboration uses, at higher energies [41, 83], a considerably wider multiplicity cut, namely $10-40 \%$ centrality, which consequently corresponds to a considerably wider impact parameter range of $4.4<b<9.5 \mathrm{fm}$. The NA49 midcentral data[40] correspond to an impact parameter range of 5.5 to $9.1 \mathrm{fm}$. The FOPI collaboration's centrality cut on their $v_{2}$ data [84] is $\mathrm{M} 3$, corresponding to $b=5.5-7.5 \mathrm{fm}$. However, in Ref. [84], FOPI shows another cut, $b=7.5-9.5 \mathrm{fm}$ (M4), with nearly identical $v_{2}$ values as in the more central M3 cuthence, all these experimental observations suggest a "midcentral impact parameter range" of $4.6<b<9.4 \mathrm{fm}$. This range was previously used to analyze directed flow by the UrQMD hybrid model collaboration [17]. This finding suggests using that same "midcentral" impact parameter range for calculations at all beam energies, at least up to $\sqrt{s_{N N}}<100 \mathrm{GeV}$. We have checked that also a "midcentral" cut of 5 to $7 \mathrm{fm}$ yields nearly the same flow value-hence, the correct results do depend only weakly on the precise values of the impact parameter cut.

\section{A. directed flow}

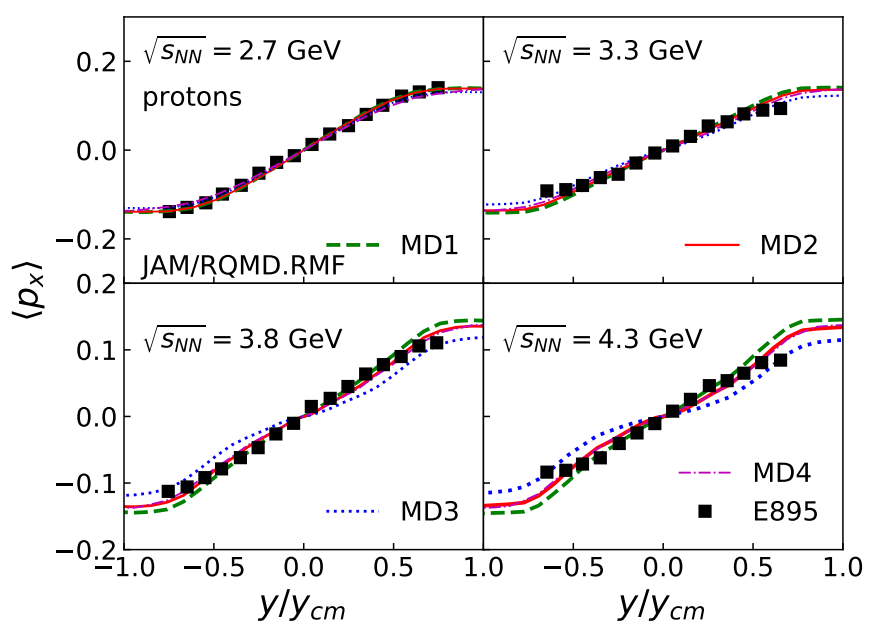

FIG. 3. Rapidity dependence of proton sideward flow $\left\langle p_{x}\right\rangle$ in midcentral $\mathrm{Au}+\mathrm{Au}$ collision at $\sqrt{s_{N N}}=2.7,3.3,3.8$ and $4.3 \mathrm{GeV}$ $\left(E_{\text {lab }}=1.85,4,6,8 \mathrm{~A} \mathrm{GeV}\right)$ from the parameter set MD1 (dashed line), MD2 (solid line), MD3 (dotted line), and MD4 (dotted-dashed line) are compared with the E895 experimental data [80]. The results of MD4 are not visible because it is nearly identical to the results of MD2.

Let us first study the optical potential dependence of the sideward flow $\left\langle p_{x}\right\rangle$ by comparing the parameter sets MD1, MD2, and MD3. All of them have the same incompressibility 
$K=380 \mathrm{MeV}$ and the effective mass $m^{*} / m=0.65$, but different strengths of the optical potential above $E_{\text {lab }}>1 \mathrm{GeV}$. Figure 3 shows the rapidity dependence of the sideward flow in midcentral $\mathrm{Au}+\mathrm{Au}$ collisions at $\sqrt{s_{N N}}=2.7,3.3,3.8$ and $4.3 \mathrm{GeV}$ with different parameter set MD1, MD2, and MD3. The full squares represent the experimental data from the E895 collaboration [80]. It is seen that all parameter sets yield similar results at $\sqrt{s_{N N}}=2.7 \mathrm{GeV}$, since EoS at 2.7 $\mathrm{GeV}$ is almost the same among MD1, MD2, and MD3, due to the constraints from experiments. As the beam energy increases, the difference among EoS becomes visible indicating the sensitivity of the sideward flow to the optical potential. We note that the results from the set MD4 is identical to the results from MD2 indicating that directed flow data is insensitive to the stiffness of the EoS.

To obtain free protons, we identify nuclear cluster based on the phase space distribution of nucleons at the end of the simulation by using a minimum distance chain procedure; two nucleons are considered to be bound in the same cluster if the relative distance and momentum between nucleons are less than $4 \mathrm{fm}$ and $0.3 \mathrm{GeV} / c$, respectively. We have found that the effects of the nuclear cluster as well as the weak decay of hyperons on the sideward flow shown in Fig. 3 are very small. However, nuclear cluster effects are large close to the target and projectile rapidities.

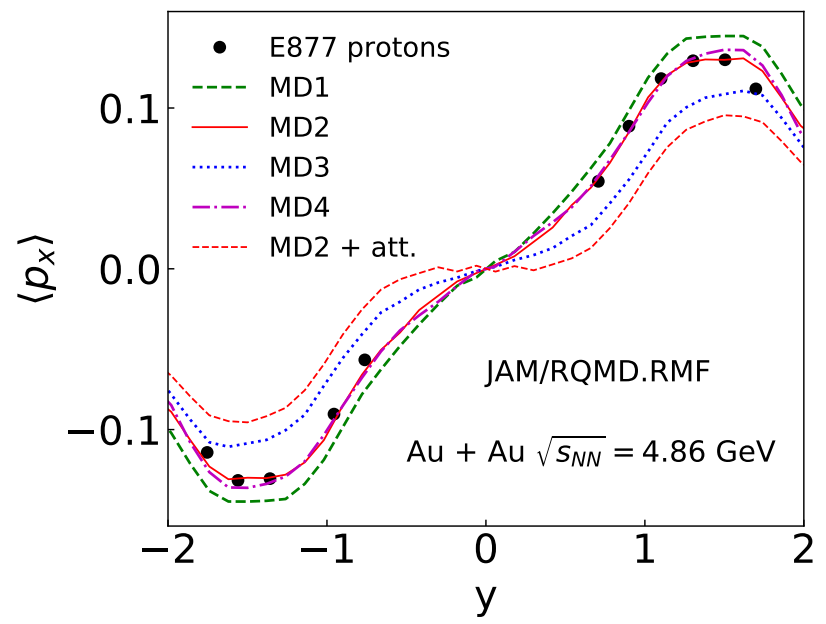

FIG. 4. Rapidity dependence of proton sideward flow $\left\langle p_{x}\right\rangle$ in midcentral $\mathrm{Au}+\mathrm{Au}$ collision at $\sqrt{s_{N N}}=4.86 \mathrm{GeV}$ from MD1 (dashed line), MD2 (solid line), MD3 (dotted line), MD4 (dotted-dashed line), and MD2 + attractive orbit are compared with the E877 experimental data [85].

We expect that the optical potential dependence of the sideward flow may become significant at higher beam energies. In Fig. 4. sideward flow from various EoS are compared for midcentral $\mathrm{Au}+\mathrm{Au}$ collision at $\sqrt{s_{N N}}=4.864 \mathrm{GeV}$. Strong sensitivity of the sideward flow to the optical potential is seen. The MD1 set overestimates the data which has a value of optical potential similar to that of the NS1 set that has almost flat optical potential as a function of kinetic energy. The MD2 set reproduces the data which has approximately twice smaller optical potential of $U_{\mathrm{opt}} \approx 30 \mathrm{MeV}$ than that of the set MD1 at $E_{\text {lab }}=10 \mathrm{GeV}$. However, the set MD4 $(K=210 \mathrm{MeV}$, $m^{*} / m=0.83$ ) can also fit the data with the optical potential $U_{\mathrm{opt}} \approx 70-90 \mathrm{MeV}$. Thus, this analysis shows that effective mass parameter and the optical potential correlate to each other; smaller effective mass needs smaller optical potential to reproduce the sideward flow data. Therefore, determination of the optical potential by experiments should give important constraint on the information about the properties of excited hadronic matter.

The phase transition to a quark-gluon plasma is connected to the softening of the EoS, and the signal may be observed in the directed flow; the slope of proton directed flow at midrapidity becomes negative [34]. The effects of the softening of the EoS can be efficiently simulated by selecting attractive orbit in every two-body scattering in a microscopic transport simulation [38, 39]. The result of MD2 + attractive orbit calculation is also plotted in Fig. 4 The rapidity dependence of the directed flow for this calculation is similar to the prediction by the hydrodynamic calculation in Ref. [34], and the slope at mid-rapidity becomes negative. However, experimental data do not indicate such softening of the EoS at $\sqrt{s_{N N}}=4.86$ $\mathrm{GeV}$. See Ref. [86] for the directed flow at mid-rapidity from the E877 collaboration, which shows positive slope for protons.

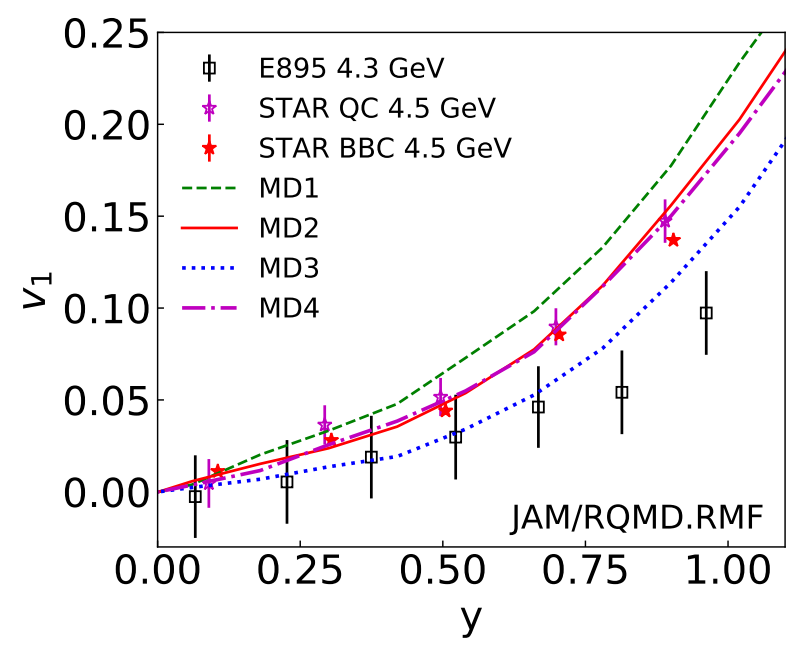

FIG. 5. Rapidity dependence of proton directed flow $v_{1}$ in midcentral $\mathrm{Au}+\mathrm{Au}$ collision at $\sqrt{s_{N N}}=4.5 \mathrm{GeV}$ from MD1 (dashed line), MD2 (solid line), MD3 (dotted line), and MD4 (dotted-dashed line) are compared with the E895 [80] and STAR data [82]. The momentum cut $0.4<p_{T}<2.0 \mathrm{GeV}$ is imposed, and only 'free' protons are selected in $v_{1}$ after nuclear coalescence.

Figure 5 shows the rapidity dependence of the directed flow $v_{1}=\left\langle p_{x} / p_{T}\right\rangle$ in midcentral $\mathrm{Au}+\mathrm{Au}$ collisions at $\sqrt{s_{N N}}=4.5 \mathrm{GeV}$ from RQMD.RMF simulations with different parameter sets, which are compared with the STAR preliminary data [82] and E895 data [80]. It is also seen that directed flow is sensitive to the EoS. The results of the MD2 and MD4 parameter set, which fit the E895 sideward flow data, are consistent with the STAR data. However, MD2 and MD4 overestimate the E895 directed flow data. We note that this discrepancy has been already discussed within three-fluid dy- 
namics (3FD) simulations [36]. 3FD reproduces the sideward flow $\left\langle p_{x}\right\rangle$ at AGS energies, while the agreement of the calculated directed flow $v_{1}$ with the E895 data is worse than the calculated sideward flow. It seems that STAR FXT data may clarify the inconsistency of the old AGS data.

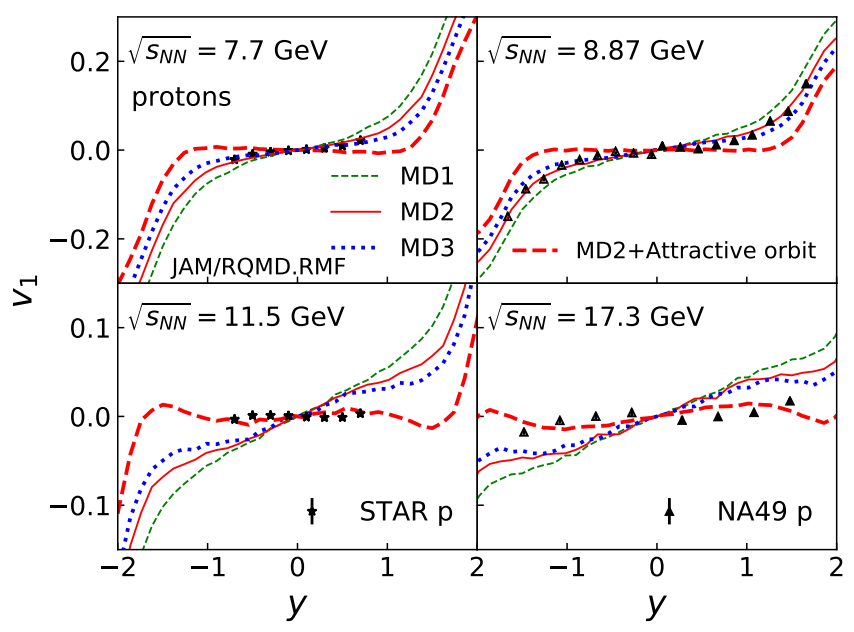

FIG. 6. Rapidity dependence of proton directed flow $v_{1}$ in midcentral $\mathrm{Au}+\mathrm{Au}$ at $\sqrt{s_{N N}}=7.7,11.5$ and $\mathrm{Pb}+\mathrm{Pb}$ collisions at 8.87, 17.3 GeV from MD1 (dashed line), MD2 (solid line), MD3 (dotted line), and MD2 + attractive orbit simulations (thick dashed line) are compared with the NA49 [40] and STAR experimental data [41].

Let us look at the directed flow at higher beam energies. Figure 6 compares the rapidity dependence of the proton directed flow from the RQMD.RMF calculations in midcentral $\mathrm{Au}+\mathrm{Au}$ at $\sqrt{s_{N N}}=7.7,11.5 \mathrm{GeV}$ and $\mathrm{Pb}+\mathrm{Pb}$ at $\sqrt{s_{N N}}=$ 8.87, $17.3 \mathrm{GeV}$ with STAR [41] and NA49 data [40]. The RQMD.RMF result with the EoS parameter set MD2 is in good agreement with the data at $\sqrt{s_{N N}}=7.7 \mathrm{GeV}$, while its slope at midrapidity is slightly higher than that of data at $\sqrt{s_{N N}}=8.87 \mathrm{GeV}$, which indicates the onset of the softening of the EoS. This is clearly seen at $\sqrt{s_{N N}}=11.5$ and $17.3 \mathrm{GeV}$, where all calculations with the 'normal' EoS predict strong positive slope in contrast to the data which show negative slope. We note that the rapidity dependence of the directed flow from the MD4 parameter set is the same as that of the MD2 parameter set.

We also compare the results from the attractive orbit simulation with the MD2 EoS which mimics a softening of the EoS [38, 39]. As shown in Ref. [38], the pressure due to the attractive orbit is reduced as low as the one in a typical EoS with a first-order phase transition. It is seen that the attractive orbit simulations predict significant reduction of the directed flow slope, and their results are close to the data at $\sqrt{s_{N N}}=11.5$ and $17.3 \mathrm{GeV}$. On the other hand, it is inconsistent with the data at $\sqrt{s_{N N}}=7.7 \mathrm{GeV}$. Note that the proton negative flow at higher energies $\sqrt{s_{N N}}>30 \mathrm{GeV}$, where secondary interactions start after two nuclei pass through each other, can be understood by the geometrical effects [87, 88]. It is very important to notice, however, that this geometrical interpolation is not applicable at $\sqrt{s_{N N}}<30 \mathrm{GeV}$, because secondary hadronic interactions alter the dynamics during the overlapping times of the colliding nuclei as hadronization time is less than the crossing time [89]. Thus, negative proton slope at $\sqrt{s_{N N}}<20 \mathrm{GeV}$ cannot be explained by the geometrical effects. Therefore, our analysis support that the collapse of the directed flow around $\sqrt{s_{N N}} \approx 10 \mathrm{GeV}$ discovered by the experiments is an evidence of the softening of the EoS. We note that the directed flow data is in favour of the crossover EoS within the 3FD calculations [35, 36, 90].

\section{B. Elliptic flow}

We now examine the beam energy dependence of the elliptic flow $v_{2}=\langle\cos 2 \phi\rangle$. Figure 7 displays the beam en-

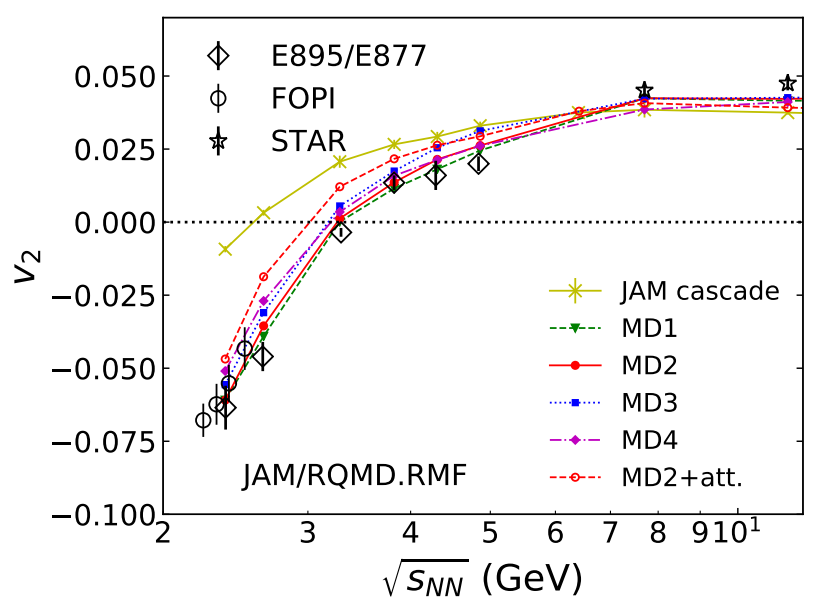

FIG. 7. Beam energy dependence of elliptic flow $v_{2}$ of proton $\left(\sqrt{s_{N N}}<5 \mathrm{GeV}\right)$ and charged hadron $\left(\sqrt{s_{N N}}>5 \mathrm{GeV}\right)$ in midcentral $\mathrm{Au}+\mathrm{Au}$ collision from MD1 (triangles), MD2 (circles), MD3 (squares), MD4 (diamond), and MD2 + attractive orbit (open circles) are compared with the experimental data FOPI [84], E895/E877 [81] and, STAR [83]. The STAR data [83] for $v_{2}$ are for charged hadrons.

ergy dependence of the elliptic flow at midrapidity in midcentral $\mathrm{Au}+\mathrm{Au}$ collisions at $\sqrt{s_{N N}}<5$ for protons, and at $\sqrt{s_{N N}}>6 \mathrm{GeV}$ for charged hadrons. Experimental data for the elliptic flow is consistent with the strong repulsive interactions in RQMD.RMF with the MD1 and MD2 parameter set, which generate strong out-of-plane emission (squeezeout), while the parameter set MD3 which has weak optical potential predicts less out-of-plane emission. The soft EoS MD4 generates weaker elliptic flow at lower beam energies. Thus, elliptic flow data exclude very weak optical potential and a soft EoS at low energies.

If there is a first-order phase transition, out-of-plane emission is suppressed, and enhancement of $v_{2}$ and $v_{4}$ is predicted within the cascade model with modified scattering style [91, 92]. To see such softening effects in our approach, we plot in Fig. 7 7the results from MD2 parameter set with attractive orbit simulation. MD2 with attractive orbit simulations yield less out-of-plane emission at AGS energies, while they 
do not change the elliptic flow much at SPS energies. Experimental data for elliptic flow, however, do not support the softening of the EoS at AGS energies. There is no data between $5<\sqrt{s_{N N}}<7.7 \mathrm{GeV}$. A new data at $\sqrt{s_{N N}} \approx 6$ $\mathrm{GeV}$ should provide further confirmation about the EoS.

Our hadronic approach predicts less elliptic flow at $\sqrt{s_{N N}}=11.5 \mathrm{GeV}$. The elliptic flow at higher energies increases due to strong in-plane emission, which is consistent with hydrodynamical calculations [18, 23], and microscopic transport models with partonic phase [93-95].

\section{SUMMARY}

We have extended a relativistic quantum molecular dynamics model based on the relativistic mean-field theory by including momentum-dependent potentials. This approach has been implemented into the JAM transport code to study the heavy-ion collisions at high baryon density region. We found that the directed flow is very sensitive to the optical potential, and there is a correlation between the effective mass parameter at the normal nuclear matter density and the strength of the optical potential. Namely, smaller effective mass requires smaller optical potential to reproduce the directed flow data at $3<\sqrt{s_{N N}}<8 \mathrm{GeV}$. Thus experimental information on the optical potential from $p A$ collisions at these energy ranges will allow us to constrain EoS.

It is also shown that the beam energy dependence of the elliptic flow at mid-rapidity is well described by the same parameter set which reproduces the directed flow data. On the other hand, stiff EoS is required to describe the strong squeeze-out at lower beam energies of $\sqrt{s_{N N}}<3 \mathrm{GeV}$, which is consistent with the transport calculations within the Skyrme type potential in Refs. [30, 70, 81, 84, 96] and the RBUU calculations [62, 63]. Within the non-relativistic QMD models with the non-relativistic Skyrme potentials, the kaon production at the Bevalac and at GSI's SiS18 was studied by Fuchs, Aichelin, and Hartnack, et al. [97--100] They suggest that soft EoS with $K=210 \mathrm{MeV}$ can be extracted from the analysis of the data at beam energies below $E_{\text {lab }}=2 \mathrm{AGeV}$. Also, $v_{1}$ and $v_{2}$ are consistent-within the non-relativistic IQMD model with a non-relativistic soft EoS [101, 102]. Hence, the claim is that the EoS extracted from the data at these moderate energies, $E_{\text {lab }}<2 \mathrm{AGeV}$, is soft; with $K=210 \mathrm{MeV}$, independent of input parameters which are not precisely known. Our results are quantitatively not consistent with these IQMD results [97--102]. The present paper, which does not investigate kaon yields at $E_{\text {lab }}<2 \mathrm{AGeV}$, focusses on baryon flow. Standard free-space elastic and inelastic scattering cross sections are used, without in-medium modifications, and without change of meson properties. Non-relativistic codes do not have different relativistic transformation laws for scalar effective mass attraction and vector repulsion. Hence, this systematic difference may the origin for the difference between non-relativistic IQMD and relativistic codes RQMD.RMF.

Our approach reproduces the directed and the elliptic flow data at $2.3<\sqrt{s_{N N}}<8 \mathrm{GeV}$ simultaneously with the pa- rameter set MD2. In contrast, this approach does not describe the collapse of the proton directed flow at $8<\sqrt{s_{N N}}<20$ $\mathrm{GeV}$ unless taking into account the effects of a softening of the EoS. We simulate effectively a softening of the EoS by imposing attractive orbit at each two-body collision. This method provides a good description of the directed flow at $\sqrt{s_{N N}}>8 \mathrm{GeV}$. However, we still cannot explain the beam energy dependence of the directed flow in a single consistent framework. We note that most of the theoretical calculations predict the collapse of the directed flow below $\sqrt{s_{N N}} \approx 6$ $\mathrm{GeV}$. Thus, it remains to be understood why softening is seen at $10<\sqrt{s_{N N}}<20 \mathrm{GeV}$, if the collapse of the directed flow is certainly due to the softening of the EoS. Furthermore, it is still premature to make an unambiguous conclusion that the collapse of the directed flow is a signature of a first-order phase transition. Theoretically, mean-field approach is a favored method to study EoS dependence in a dynamical simulation. As a future work, it would be interesting to simulate chiral phase transition within the RQMD approach based on chiral mean field model [103].

\section{ACKNOWLEDGMENTS}

We thank W. Cassing, C. Hartnack and J. Steinheimer for valuable comments on the manuscript, and D.Keane and Y. Wu for clarifying the STAR and E896 data. This work was supported in part by the Grants-in-Aid for Scientific Research from JSPS (JP17K05448 and JP19K03833). H. S. thanks the Walter Greiner Gesellschaft for the Judah M. Eisenberg Laureatus Professur. Computational resources have been provided by GSI, Darmstadt, and LOEWE CSC, Goethe Universität Frankfurt.

\section{Appendix A: Equation of motion}

In RQMD, scalar density and vector potential are computed by using the Gaussian $\rho_{i j}$ :

$$
\rho_{s_{i}}=\sum_{j \neq i} f_{j} \rho_{i j}, \quad V_{i}^{\mu}=C_{v} B_{i} \sum_{j \neq i} B_{j} u_{j}^{\mu} \rho_{i j}
$$

where $f_{j}=\frac{m_{j}}{p_{j}^{0}}, u_{j}^{\mu}=\frac{p_{j}^{\mu}}{p_{j}^{0}}$, and $B_{j}$ is a baryon number of particle $j$. The Gaussian $\rho_{i j}$ is given by

$$
\rho_{i j}=\frac{\gamma_{i j}}{(2 \pi L)^{3 / 2}} \exp \left[\frac{q_{T, i j}^{2}}{2 L}\right] .
$$

The equations of motion Eq. 21] can be computed as

$$
\begin{aligned}
\dot{\boldsymbol{r}}_{i}=\frac{\boldsymbol{p}_{i}^{*}}{p_{i}^{* 0}}+\sum_{j \neq i}\left[D_{i j} \frac{\partial \rho_{i j}}{\partial \boldsymbol{p}_{i}}+D_{j i} \frac{\partial \rho_{j i}}{\partial \boldsymbol{p}_{i}}\right. \\
\left.+\left(D_{j} \frac{\partial f_{i}}{\partial \boldsymbol{p}_{i}}+A_{j}^{\mu} \frac{\partial u_{i \mu}}{\partial \boldsymbol{p}_{i}}\right) \rho_{j i}\right] \\
\dot{\boldsymbol{p}}_{i}=-\sum_{j \neq i}\left[D_{i j} \frac{\partial \rho_{i j}}{\partial \boldsymbol{r}_{i}}+D_{j i} \frac{\partial \rho_{j i}}{\partial \boldsymbol{r}_{i}}\right]
\end{aligned}
$$


where

$$
\begin{aligned}
D_{i j} & =D_{i} f_{j}+A_{i j}^{\mu} u_{j \mu}, \\
D_{i} & =\frac{m_{i}^{*}}{p_{i}^{* 0}} \frac{\partial S_{i}}{\partial \rho_{s i}}, \\
A_{i j}^{\mu} & =C_{v} B_{i} B_{j} v_{i}^{* \mu} .
\end{aligned}
$$

When the two-body or overall center-of-mass frame is used to define $q_{T, i j}$, the Gaussian $\rho_{i j}$ is symmetric: $\rho_{i j}=\rho_{j i}$. For the momentum-dependent potentials, we need derivatives of an additional terms:

$$
\bar{\rho}_{i j}=D\left(\boldsymbol{p}_{i j}^{2}\right) \rho_{i j}, \quad D\left(\boldsymbol{p}_{i j}^{2}\right)=\frac{\bar{C}}{1+\boldsymbol{p}_{i j}^{2} / \Lambda^{2}} .
$$

In the case of the nonlinear $\sigma$ field

$$
m_{\sigma}^{2} \sigma_{i}+g_{2} \sigma_{i}^{2}+g_{3} \sigma_{i}^{3}=g_{s} \rho_{s i}
$$

The derivatives $\partial S_{i} / \partial \rho_{s i}$ can be obtained by

$$
\frac{\partial S_{i}}{\partial \rho_{s i}}=-g_{s} \frac{\partial \sigma_{i}}{\partial \rho_{s i}}=\frac{-g_{s}^{2}}{m_{\sigma}^{2}+2 g_{2} \sigma_{i}+3 g_{3} \sigma_{i}^{2}}
$$

[1] S. Singha, P. Shanmuganathan and D. Keane, Adv. High Energy Phys. 2016, 2836989 (2016).

[2] J. Adam et al. [STAR Collaboration], arXiv:1903.11778 [nuclex].

[3] L. Turko [NA61/SHINE Collaboration], Universe 4, no. 3, 52 (2018); Katarzyna Grebieszkow [NA61/SHINE Collaboration], arXiv:1904.03165 [nucl-ex].

[4] G. Odyniec, EPJ Web Conf. 95, 03027 (2015).

[5] T. Ablyazimov et al. [CBM Collaboration], Eur. Phys. J. A 53, no. 3, 60 (2017).

[6] C. Sturm, B. Sharkov and H. Stoecker, Nucl. Phys. A 834, 682c (2010).

[7] V. Kekelidze, A. Kovalenko, R. Lednicky, V. Matveev, I. Meshkov, A. Sorin, and G. Trubnikov, Nucl. Phys. A 956, 846 (2016).

[8] H. Sako et al., [J-PARC Heavy-Ion Collaboration], Nucl. Phys. A 9311158 (2014); Nucl. Phys. A 956, 850 (2016).

[9] H. Sorge, H. Stoecker and W. Greiner, Annals Phys. 192, 266 (1989).

[10] H. Sorge, Phys. Rev. C 52, 3291 (1995).

[11] S. A. Bass et al., Prog. Part. Nucl. Phys. 41, 255 (1998).

[12] M. Bleicher et al., J. Phys. G 25, 1859 (1999).

[13] W. Cassing and E. L. Bratkovskaya, Phys. Rept. 308, 65 (1999).

[14] O. Buss et al., Phys. Rept. 512, 1 (2012).

[15] Y. B. Ivanov and A. A. Soldatov, Phys. Rev. C 101, no. 2, 024915 (2020); Phys. Rev. C 98, no. 1, 014906 (2018); Phys. Rev. C 97, no. 2, 024908 (2018).

[16] H. Petersen, J. Steinheimer, G. Burau, M. Bleicher and H. Stoecker, Phys. Rev. C 78, 044901 (2008).

[17] J. Steinheimer, J. Auvinen, H. Petersen, M. Bleicher and H. Stoecker, Phys. Rev. C 89, no.5, 054913 (2014).

[18] I. A. Karpenko, P. Huovinen, H. Petersen and M. Bleicher, Phys. Rev. C 91, no. 6, 064901 (2015).

[19] P. Batyuk et al., Phys. Rev. C 94, 044917 (2016).

[20] C. Shen and B. Schenke, Phys. Rev. C 97, no. 2, 024907 (2018).

[21] G. S. Denicol, C. Gale, S. Jeon, A. Monnai, B. Schenke and C. Shen, Phys. Rev. C 98, no. 3, 034916 (2018).

[22] Y. Akamatsu et al., Phys. Rev. C 98, no. 2, 024909 (2018).

[23] C. Shen, S. Alzhrani, |arXiv:2003.05852 [nucl-th]].

[24] H. Stoecker, J. A. Maruhn and W. Greiner, Phys. Rev. Lett. 44, 725 (1980).

[25] H. Stoecker, L. P. Csernai, G. Graebner, G. Buchwald, H. Kruse, R. Y. Cusson, J. A. Maruhn and W. Greiner, Phys.
Rev. C 25, 1873 (1982).

[26] G. Buchwald, G. Graebner, J. Theis, J. Maruhn, W. Greiner, H. Stoecker, K. A. Frankel and M. Gyulassy, Phys. Rev. C 28, 2349 (1983).

[27] H. Stoecker and W. Greiner, Phys. Rept. 137, 277 (1986).

[28] C. Hartnack, J. Aichelin, H. Stoecker and W. Greiner, Phys. Lett. B 336, 131 (1994).

[29] J.-Y. Ollitrault, Phys. Rev. D 46 (1992) 229.

[30] P. Danielewicz, R. Lacey and W. G. Lynch, Science 298, 1592 (2002).

[31] H. Stoecker, Nucl. Phys. A 750, 121 (2005).

[32] D. H. Rischke, Y. Pursun, J. A. Maruhn, H. Stoecker and W. Greiner, Acta Phys. Hung. A 1, 309 (1995).

[33] J. Brachmann, S. Soff, A. Dumitru, H. Stoecker, J. A. Maruhn, W. Greiner, L. V. Bravina and D. H. Rischke, Phys. Rev. C 61, 024909 (2000).

[34] L. P. Csernai and D. Rohrich, Phys. Lett. B 458, 454 (1999).

[35] Y. B. Ivanov and A. A. Soldatov, Phys. Rev. C 91, no. 2, 024915 (2015).

[36] Y. B. Ivanov and A. A. Soldatov, Eur. Phys. J. A 52, no. 1, 10 (2016).

[37] B. A. Li and C. M. Ko, Phys. Rev. C 58, 1382 (1998).

[38] Y. Nara, H. Niemi, A. Ohnishi and H. Stoecker, Phys. Rev. C 94, no. 3, 034906 (2016).

[39] Y. Nara, H. Niemi, J. Steinheimer and H. Stoecker, Phys. Lett. B 769, 543 (2017).

[40] C. Alt et al. [NA49 Collaboration], Phys. Rev. C 68, 034903 (2003).

[41] L. Adamczyk et al. [STAR Collaboration], Phys. Rev. Lett. 112, no. 16, 162301 (2014).

[42] L. Adamczyk et al. [STAR Collaboration], Phys. Rev. Lett. 120, no. 6, 062301 (2018).

[43] J. Xu et al., Phys. Rev. C 93, no. 4, 044609 (2016); Y. X. Zhang et al., Phys. Rev. C 97, no. 3, 034625 (2018); A. Ono et al., Phys. Rev. C 100 (2019) no.4, 044617.

[44] B. Blattel, V. Koch, W. Cassing and U. Mosel, Phys. Rev. C 38, 1767 (1988). B. Blaettel, V. Koch and U. Mosel, Rept. Prog. Phys. 56, 1 (1993).

[45] C. M. Ko, Q. Li and R. C. Wang, Phys. Rev. Lett. 59, 1084 (1987); Q. Li, J. Q. Wu and C. M. Ko, Phys. Rev. C 39, 849 (1989); H. T. Elze, M. Gyulassy, D. Vasak, H. Heinz, H. Stoecker and W. Greiner, Mod. Phys. Lett. A 2, 451 (1987).

[46] C. M. Ko and Q. Li, Phys. Rev. C 37, 2270 (1988).

[47] W. Cassing and U. Mosel, Prog. Part. Nucl. Phys. 25, 235 (1990). 
[48] C. Fuchs and H. H. Wolter, Nucl. Phys. A 589, 732 (1995).

[49] J. Aichelin and H. Stoecker, Phys. Lett. B 176, 14 (1986); J. Aichelin, Phys. Rept. 202, 233 (1991).

[50] J. Aichelin, E. Bratkovskaya, A. Le Févre, V. Kireyeu, V. Kolesnikov, Y. Leifels, V. Voronyuk and G. Coci, Phys. Rev. C 101, no.4, 044905 (2020).

[51] T. Maruyama, S. W. Huang, N. Ohtsuka, G. Q. Li, A. Faessler and J. Aichelin, Nucl. Phys. A 534, 720 (1991).

[52] T. Maruyama, K. Niita, T. Maruyama, S. Chiba, Y. Nakahara and A. Iwamoto, Prog. Theor. Phys. 96, 263 (1996).

[53] D. Mancusi, K. Niita, T. Maruyama and L. Sihver, Phys. Rev. C 79, 014614 (2009).

[54] M. Isse, A. Ohnishi, N. Otuka, P. K. Sahu and Y. Nara, Phys. Rev. C 72, 064908 (2005).

[55] C. Fuchs, E. Lehmann, L. Sehn, F. Scholz, T. Kubo, J. Zipprich and A. Faessler, Nucl. Phys. A 603, 471 (1996).

[56] Y. Nara and H. Stoecker, Phys. Rev. C 100, no. 5, 054902 (2019).

[57] Y. Nara, N. Otuka, A. Ohnishi, K. Niita and S. Chiba, Phys. Rev. C 61, 024901 (2000).

[58] J. Aichelin, A. Rosenhauer, G. Peilert, H. Stoecker and W. Greiner, Phys. Rev. Lett. 58, 1926 (1987).

[59] G. M. Welke, M. Prakash, T. T. S. Kuo, S. Das Gupta and C. Gale, Phys. Rev. C 38, 2101 (1988); C. Gale, G. M. Welke, M. Prakash, S. J. Lee and S. Das Gupta, Phys. Rev. C 41, 1545 (1990).

[60] K. Weber, B. Blaettel, W. Cassing, H. C. Doenges, V. Koch, A. Lang and U. Mosel, Nucl. Phys. A 539, 713 (1992).

[61] T. Maruyama, B. Blaettel, W. Cassing, A. Lang, U. Mosel and K. Weber, Phys. Lett. B 297, 228 (1992).

[62] T. Maruyama, W. Cassing, U. Mosel, S. Teis and K. Weber, Nucl. Phys. A 573, 653 (1994).

[63] P. K. Sahu, A. Hombach, W. Cassing and U. Mosel, Nucl. Phys. A 640, 493 (1998); P. K. Sahu, W. Cassing, U. Mosel and A. Ohnishi, Nucl. Phys. A 672, 376 (2000); P. K. Sahu and W. Cassing, Nucl. Phys. A 712, 357 (2002).

[64] J. Boguta and A.R. Bodmer, Nucl. Phys. A 292, 413 (1977).

[65] S. Hama, B. C. Clark, E. D. Cooper, H. S. Sherif and R. L. Mercer, Phys. Rev. C 41, 2737 (1990).

[66] H. Feldmeier and J. Lindner, Z. Phys. A 341, 83 (1991).

[67] P. Danielewicz, Nucl. Phys. A 673, 375 (2000).

[68] J. I. Kapusta and C. Gale, "Finite-temperature field theory: Principles and applications," Cambridge University Press (2011).

[69] G. Rai et al. [E895 Collaboration], Nucl. Phys. A 661, 162 (1999).

[70] P. Hillmann, J. Steinheimer and M. Bleicher, J. Phys. G 45, no. 8, 085101 (2018).

[71] A. Komar, Phys. Rev. D 18, 1881 (1978); Phys. Rev. D 18, 1887 (1978); Phys. Rev. D 18, 3617 (1978).

[72] R. Marty and J. Aichelin, Phys. Rev. C 87, no. 3, 034912 (2013).

[73] D. Oliinychenko and H. Petersen, Phys. Rev. C 93, no. 3, 034905 (2016).

[74] G. Q. Li, C. M. Ko and G. E. Brown, Phys. Rev. Lett. 75, 4007 (1995); Nucl. Phys. A 606, 568 (1996).

[75] A. B. Larionov, O. Buss, K. Gallmeister and U. Mosel, Phys.
Rev. C 76, 044909 (2007).

[76] W. Cassing, A. Palmese, P. Moreau and E. L. Bratkovskaya, Phys. Rev. C 93, 014902 (2016); A. Palmese, W. Cassing, E. Seifert, T. Steinert, P. Moreau and E. L. Bratkovskaya, Phys. Rev. C 94, no. 4, 044912 (2016).

[77] K. Weber, B. Blattel, V. Koch, A. Lang, W. Cassing and U. Mosel, Nucl. Phys. A 515, 747 (1990).

[78] T. Hirano and Y. Nara, PTEP 2012, 01 A203 (2012).

[79] D. Oliinychenko and H. Petersen, J. Phys. G 44, no. 3, 034001 (2017).

[80] H. Liu et al. [E895 Collaboration], Phys. Rev. Lett. 84, 5488 (2000);

[81] C. Pinkenburg et al. [E895 Collaboration], Phys. Rev. Lett. 83, 1295 (1999).

[82] Y. Wu [STAR Collaboration], Nucl. Phys. A 982, 899 (2019).

[83] L. Adamczyk et al. [STAR Collaboration], Phys. Rev. C 86, 054908 (2012).

[84] A. Andronic et al. [FOPI Collaboration], Phys. Lett. B 612, 173 (2005)

[85] J. Barrette et al. [E877 Collaboration], Phys. Rev. C 56, 3254 (1997).

[86] J. Barrette et al. [E877 Collaboration], Phys. Rev. C 55, 1420 (1997) Erratum: [Phys. Rev. C 56, 2336 (1997)].

[87] R. J. M. Snellings, H. Sorge, S. A. Voloshin, F. Q. Wang and N. Xu, Phys. Rev. Lett. 84, 2803 (2000).

[88] C. Zhang, J. Chen, X. Luo, F. Liu and Y. Nara, Phys. Rev. C 97, no. 6, 064913 (2018).

[89] H. Sorge, Phys. Lett. B 402, 251 (1997).

[90] V. P. Konchakovski, W. Cassing, Y. B. Ivanov and V. D. Toneev, Phys. Rev. C 90, no. 1, 014903 (2014).

[91] Y. Nara, H. Niemi, A. Ohnishi, J. Steinheimer, X. Luo and H. Stoecker, Eur. Phys. J. A 54, no. 2, 18 (2018).

[92] Y. Nara, J. Steinheimer and H. Stoecker, Eur. Phys. J. A 54, no. 11, 188 (2018).

[93] Z. W. Lin and C. M. Ko, Phys. Rev. C 65, 034904 (2002).

[94] V. P. Konchakovski, E. L. Bratkovskaya, W. Cassing, V. D. Toneev and V. Voronyuk, Phys. Rev. C 85, 011902 (2012).

[95] V. P. Konchakovski, E. L. Bratkovskaya, W. Cassing, V. D. Toneev, S. A. Voloshin and V. Voronyuk, Phys. Rev. C 85, 044922 (2012).

[96] A. Larionov, W. Cassing, C. Greiner and U. Mosel, Phys. Rev. C 62, 064611 (2000).

[97] C. T. Sturm et al. [KAOS], Phys. Rev. Lett. 86, 39-42 (2001).

[98] C. Fuchs, A. Faessler, E. Zabrodin and Y. M. Zheng, Phys. Rev. Lett. 86, 1974-1977 (2001).

[99] C. Hartnack, H. Oeschler and J. Aichelin, Phys. Rev. Lett. 96, 012302 (2006).

[100] C. Hartnack, H. Oeschler, Y. Leifels, E. L. Bratkovskaya and J. Aichelin, Phys. Rept. 510, 119-200 (2012).

[101] W. Reisdorf et al. [FOPI], Nucl. Phys. A 876, 1-60 (2012).

[102] A. Le Fvre, Y. Leifels, W. Reisdorf, J. Aichelin and C. Hartnack, Nucl. Phys. A 945, 112-133 (2016).

[103] A. Motornenko, J. Steinheimer, V. Vovchenko, S. Schramm and H. Stoecker, Phys. Rev. C 101, no. 3, 034904 (2020). 